\title{
Uso del Haptic Paddle con aprendizaje basado en proyectos
}

\author{
Juan M. Gandarias, Antonio J. Muñoz-Ramírez y Jesús M. Gómez-de-Gabriel \\ Dto. de Ingeniería de Sistemas y Automática \\ Escuela de Ingenierías Industriales \\ Universidad de Málaga \\ jesus.gomez@uma.es
}

\begin{abstract}
Resumen
En este trabajo se presenta la experiencia de la utilización docente de un dispositivos háptico desarrollado como una nueva versión del Haptic Paddle, creado en la Universidad de Stanford a mediados de los 90. Se trata de un dispositivo educativo de bajo coste y simple que puede ser ensamblado y programado por los estudiantes, y que se usó para enseñanza de dinámica de sistemas. El diseño realizado usa una electrónica completamente off the shelf, rodamientos y tornillería métrica estándar y piezas fabricadas mediante impresión 3D. En este trabajo se presenta este dispositivo junto con la experiencia de su utilización docente, mediante aprendizaje basado en proyectos, en una asignatura de máster de ingeniería mecatrónica. Se trata de la primera experiencia con un total de ocho kits de haptic paddle en la asignatura de Teleoperación y Telerrobótica, junto con aprendizaje basado en proyectos (ABP) y el uso de lenguajes de modelado. Se describen la organización y el desarrollo de las sesiones de prácticas con conclusiones sobre la adecuación del los dispositivos y métodos utilizados.
\end{abstract}

Palabras clave: Educación, Ingeniería basada en modelos, Aprendizaje basado en proyectos, Haptics, Teleoperación, Simulink.

\section{INTRODUCCIÓN}

Las nuevas tendencias en la enseñanza de la ingeniería vienen ligadas a conceptos como aprendizaje colaborativo (AC), aprendizaje basado en problemas/proyectos (ABP) o el aprendizaje autónomo, entre otros. La incorporación de estos conceptos en la formación universitaria forma parte de una metodología integradora que aproxima al estudiante a su futura vida profesional.

El uso de equipos de prácticas de bajo coste se ha visto impulsado en los últimos años en distintas ramas de la ingeniería como la robótica [6] y la telerrobótica [13] gracias a la aparición de nuevas tecnologías basadas en software y hardware libres como el uso de impresoras 3D o plataformas, co- mo Arduino. En este sentido se pueden encontrar un gran número de trabajos en los que se utilizan equipos de prácticas con distintas metodologías docentes. En [7], por ejemplo, se describe el uso de robots móviles LEGO NXT con LabVIEW en asignaturas de mecatrónica.

Dentro del uso de equipos de prácticas de robótica y la aplicación de distintas metodologías docentes, se pueden encontrar trabajos en los que se aplica el uso de programación basada en lenguajes de modelado de dominio específico $[6,8,14]$. En otros trabajos se aplica la programación basada en modelos de dominio específico con el fin de aliviar la carga del docente $[1,2,3]$.

Por otro lado, existen equipos de prácticas basados en dispositivos hápticos. Entre ellos destaca en el uso de las paletas hápticas o haptic paddle [15]. El haptic paddle es un dispositivo didáctico de realimentación de fuerzas que dispone de un único grado de libertad, actuado mediante un motor de corriente continua, con un sensor de posición angular y un microcontrolador, generalmente, basado en Arduino. Este tipo de dispositivos ha sido ampliamente probado en labores docentes de robótica, háptica y teleoperación, modelado y simulación de sistemas y programación de entornos virtuales $[10,16,9,4,13,5]$.

Si bien existen diferencias entre las distintas versiones, todas las paletas hápticas comparten un a serie de características principales :

- Un grado de libertad: Lo que reduce su coste y complejidad

- Realimentación de fuerzas de tipo impedancia: el dispositivo usa como entradas el par, y devuelve su posición. La fuerza que se presenta al usuario se calcula en base al desplazamiento.

- Motor eléctrico de corriente continua.

- Interfaz de usuario de tipo palanca.

- Control mediante un microcontrolador embebido en tiempo real. 


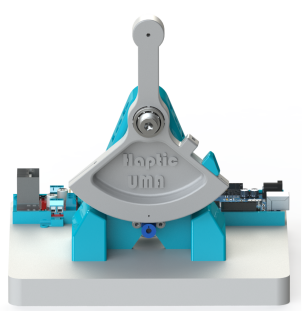

(a) Vista frontal

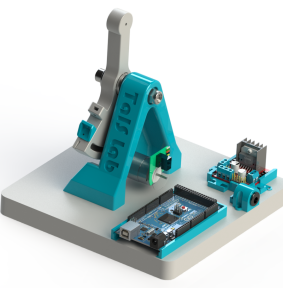

(b) Vista trasera
Figura 1: Vista frontal y trasera de los equipos de prácticas utilizados durante el curso 2016/2017

Este artículo presenta las experiencias del uso de la metodología ABP y de programación de basada en lenguajes de modelado de dominio específico (simulink) con los haptics paddle. Asimismo, se hace una valoración de los resultados obtenidos en base a los modelos docentes aplicados.

El desarrollo e implementación de los haptic paddle se ha realizado en el TaISLab (Teleoperation and Interactive Systems Laboratory) del Departamento de Ingeniería de Sistemas y Automática de la Universidad de Málaga [19]. Asimismo, se ha creado un repositorio Github [18] donde se encuentran los archivos y programas relacionados con el proyecto.

El artículo se estructura de la siguiente manera. En la sección 2, se presentan las características de los equipos de prácticas utilizados, así como los detalles de implementación y las metodologías docentes aplicadas. En la sección 3 se describe el entorno de aplicación de los haptic paddle y las distintas técnicas de enseñanza. A continuación se detallan la experiencia y los resultados obtenidos y, por último, se describen las conclusiones.

\section{HAPTIC PADDLE DEL TAISLAB}

\subsection{Características generales}

En las figura 1 se muestra la vista lateral y frontal del dispositivo haptic paddle desarrollado para la aplicación de las metodologías docentes que se describen en este artículo.

En la figura 2 se pueden observar algunos de los equipos de prácticas que se han construído para su utilización en la asignatura de Teleoperación y Telerrobótica del Máster en Ingeniería Mecatrónica de la Universidad de Málaga.

Cada de uno de estos equipos consta de un haptic paddle construido mediante técnicas de fabricación aditivas con impresoras 3D comerciales de tecnología FDM (Modelado de Fusión por Depo-

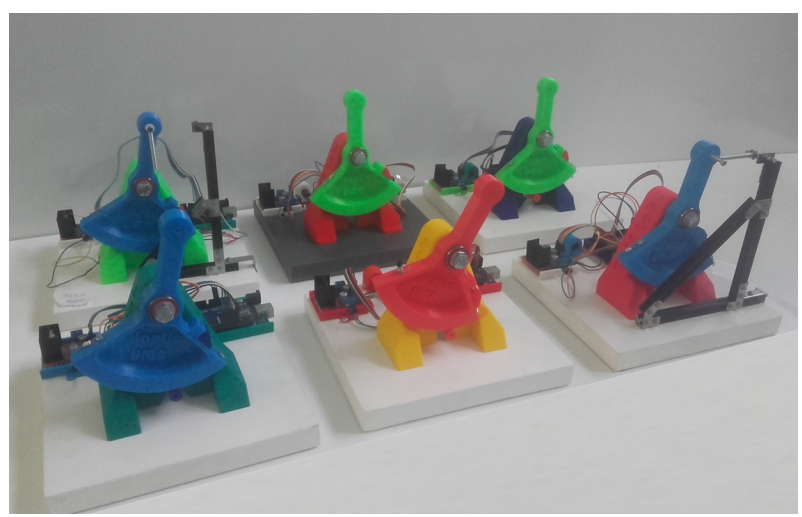

Figura 2: Equipos de prácticas utilizados durante el curso 2016/2017, junto con los dispositivos de calibración utilizados

sición), siguiendo el modelo presentado en [4], que dispone de transmisión tipo capstan, un motor de corriente continua mitsumi de la serie m25n-2 con un encoder óptico de 448 pulsos por revolución, un Arduino Mega 2560 y una etapa de potencia L298N.

\subsection{Modelado de dominio específico y ABP}

Uno de los objetivos principales que persigue el uso de estos equipos de prácticas es el uso de la programación basada en modelos de dominio específico, como es el caso de Simulink. El motivo principal es eliminar la necesidad de explicar conceptos de programación, de forma que el profesor se pueda centrar en los contenidos propios de la materia.

Para llevar a cabo las actividades de esta forma, se les ha proporcionado a los alumnos los bloques de lectura del encoder y de actuación y se les han propuesto una serie de prácticas guiadas que les sirvan para familiarizarse con la plataforma Simulink y afianzar algunos conceptos teóricos explicados con anterioridad. Finalmente, se les ha pedido que realicen un proyecto que servirá como trabajo de evaluación de su aprendizaje, siguiendo la metodología ABP.

En el ABP se crea una experiencia de aprendizaje que motiva al estudiante mediante la realización de un proyecto donde se ponen en práctica conceptos ya aprendidos pero que se muestran insuficientes para la resolución del proyecto. Se crea así la necesidad del aprendizaje de nuevos conceptos, donde el profesor posee tan sólo el papel de guía y el estudiante debe tomar un rol activo, desarrollando así capacitaciones que van a requerir en su vida profesional.

Una definición extendida de aprendizaje colabo- 
rativo (AC) recogida en [17] lo identifica con la adquisición de destrezas y actitudes que ocurren como resultado de la integración en grupo. Esto es lo deseable para la consecución de los objetivos de aprendizaje fijados en la asignatura, si bien, para que ello ocurra no basta con juntar a los estudiantes sino que es necesario que exista interactividad, sincronía y negociación, tal y como plantea [11], aportando a su vez la siguientes ventajas al estudiante: seguridad en si mismo, pensamiento crítico, solidaridad, respeto y reducción del individualismo.

El ABP es un estimulante del AC referido a pequeños grupos (dos estudiantes en este trabajo), donde los grupos reciben instrucciones del profesor para lograr el objetivo del proyecto propuesto, y los estudiantes trabajan en su consecución aprendiendo a través de la colaboración. Algunos autores como [12] usan la denominación de Aprendizaje Basado en Proyectos Colaborativo (ABPC) para este caso particular.

Todo ABP debe de cumplir con dos condicionantes: ser interesante para los estudiantes a nivel personal, y cumplir con un fin educativo. El interés de los estudiante se motiva proponiéndoles que ellos mismos creen el enunciado y objetivos del proyecto, mientras que el fin educativo se consigue definiendo los componentes y herramientas a utilizar (haptic paddle) y supervisando que los proyectos propuestos se encuentren dentro de los contenidos de la asignatura.

\section{APLICACIÓN DOCENTE}

\subsection{Asignatura}

Los haptic paddle se han utilizado en la asignatura Teleoperación y Telerrobótica del Máster en Ingeniería Mecatrónica de la Universidad de Málaga durante el segundo cuatrimestre del curso 201617. La asignatura cuenta con 14 alumnos, por lo que se han dispuesto 8 equipos de prácticas, y los alumnos han formado equipos por parejas o individuales. En la imagen de la figura 3 se observa el uso por parte de los alumnos de los dispositivos de prácticas durante la primera semana de prácticas, donde los propios alumnos efectúan el ensamblado mecánico de los dispositivos. En la figura 4 se pueden ver algunos alumnos de la asignatura y los autores.

La primera parte de la asignatura incluye temario teórico (Véase tabla 1) donde se incluye un resumen del temario.

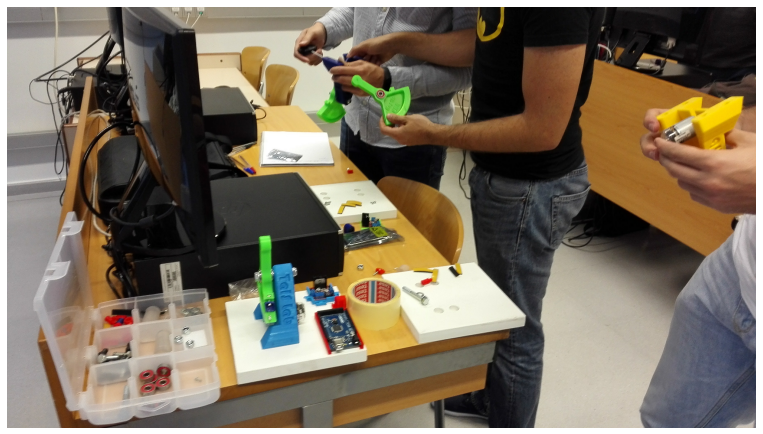

Figura 3: Montaje de los equipos por parte de los alumnos durante una sesión de prácticas

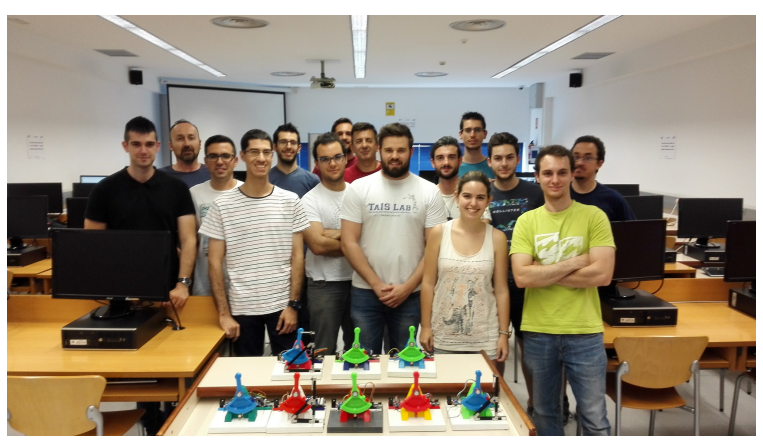

Figura 4: Alumnos de la asignatura Teleoperación y Telerrobótica del Máster en Ingeniería Mecatrónica de la Universidad de Málaga, junto a los autores de este artículo durante una sesión de prácticas con los haptic paddle

Tabla 1: Temario resumido de la asignatura de teleoperación y telerrobótica.

\begin{tabular}{|l|l|}
\hline Semana & Tema \\
\hline 1 & Introducción a la Teleoperación y la Tele- \\
& rrobótica \\
3 & Haptics \\
4 & Problemas y Métodos de Teleoperación \\
5 & Sistemas Bilaterales \\
& Retardos y métodos de evitación de los \\
6 & efectos \\
$7-12$ & Métodos telerrobóticos \\
13 & Proyecto tutorizado \\
\hline
\end{tabular}




\subsection{Conocimientos previos al uso de los equipos}

Dado que la asignatura pertenece al segundo semestre del máster, se pueden presuponer ciertos conocimientos que los alumnos ya deben haber adquirido, o bien en el primer cuatrimestre de dicho máster, o bien a lo largo del grado que hayan realizado previo a su incorporación al máster. Los alumnos, por tanto, poseen conocimientos de programación hardware en lenguajes como $\mathrm{C} / \mathrm{C}++$, que conocen el entorno de Matlab y Simulink, con los que han desarrollado prácticas previamente en otras asignaturas del máster, conocimientos técnicos de electrónica relativos al uso de microcontroladores, sensores y actuadores, así como de teoría y estrategias de control y modelado de sistemas mecatrónicos.

\subsection{Organización del programa de prácticas}

La asignatura de Teleoperación y Telerrobótica se desarrolla a lo largo de 13 semanas, de las cuales las seis últimas se han dedicado al desarrollo del proyecto de alumnos. Cada semana cuenta con dos sesiones de $1.5 \mathrm{~h}$, de las cuales la primera incluye presentaciones teóricas del profesor introduciendo los objetivos de la semana y proporcionando los métodos y materiales de trabajo, y la segunda es exclusivamente de trabajo tutorizado del alumno, tal como se resume en la tabla 2 .

\subsection{Proyectos derivados de la aplicación de la metodología ABP}

Para la realización de proyectos, los alumnos se han organizado por grupos y se les ha pedido que diseñen sus propios proyectos. Los diferentes proyectos diseñados se recogen en la tabla 3 .

Los proyectos han sido refinados con la ayuda del profesor para tener en cuenta las limitaciones de los dispositivos y del tiempo disponible para su realización.

\section{EVALUACIÓN Y RESULTADOS}

La evaluación de la asignatura se compone de la evaluación de otras prácticas realizadas durante el curso, del resultado de un cuestionario, y de la evaluación de los resultados del proyecto.

Los resultados de los proyectos son defendidos mediante una breve presentación en la sesión de evaluación, junto con una demostración de su funcionamiento. Todos los proyectos, han alcanzado los objetivos y han superado correctamente las prue- bas. No obstante existen diferencias en la complejidad del proyecto que hacen que se puntúen en mayor medida unos proyectos sobre otros.

Para evaluar de manera objetiva el efecto del uso de los haptic paddle como herramienta para el aprendizaje basado en proyectos, se ha utilizado un cuestionario con preguntas relacionadas con los dispositivos hápticos, que se formuló a alumnos del curso 2015/16 que no usaron este dispositivo, y a alumnos del curso 2016/17 que sí lo han utilizado como equipo de prácticas en su proyecto de la asignatura de Teleoperación y telerrobótica del Máster de Ingeniería Mecatrónica. No obstante, durante ambos cursos se han realizado también prácticas de otro tipo con dispositivos hápicos comerciales (Falcon) de tres grados de libertad, utilizando el lenguaje de programación LabVIEW.

El cuestionario está compuesto por trece preguntas de tipo test, con cuatro posibles respuestas en relación a los dispositivos hápticos: frecuencias de actualización, tipos de señales de entrada/salida, factores que influyen en su rendimiento, etc. Los resultados del cuestionario ofrecen una calificación media muy superior en el caso del curso 20167/17 (8.2 sobre 10) sobre el curso en el que no se utilizó (5.2 sobre 10$)$, si bien el número de sesiones dedicado a trabajar sobre el tema se ha incrementado. En la figura 5 se muestra la comparación de las calificaciones medias por pregunta.

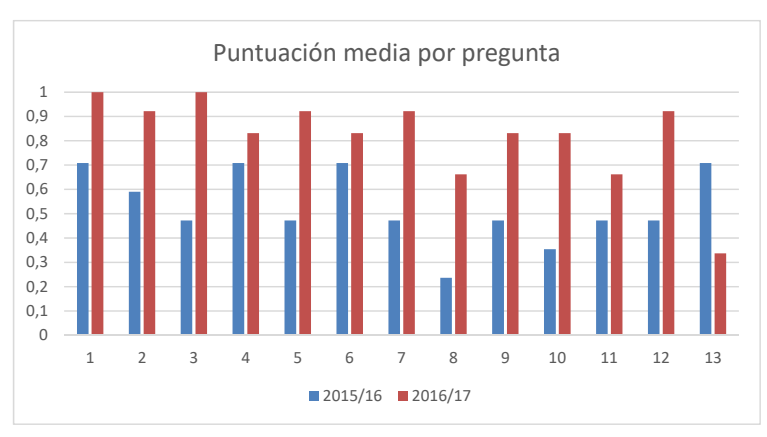

Figura 5: Resultados de la evaluación objetiva. Puntuaciones medias de cada una de las preguntas del test durante el curso en el que no se han usado los haptic paddle (2015/16) y en el que sí se han utilizado (2016/17) como plataforma para educación basada en proyectos

\section{CONCLUSIONES}

El uso de estos equipos docentes motiva en gran medida a los estudiantes y a los profesores, que junto con el uso del ABP y el permitir a los alumnos diseñar su propio proyecto aumentan dicha motivación. Esta diversidad en los proyectos debe ser supervisada por el profesor, pero aumenta la cohesión del grupo y mejora el aprendizaje co- 
Tabla 2: Organización de sesiones prácticas del asignatura de Teleoperación y Telerrobótica. Cada semana incluye dos sesiones de $1.5 \mathrm{~h}$. La primera incluye presentaciones del profesor y la segunda es exclusivamente trabajo tutorizado del alumno.

\begin{tabular}{|c|c|c|c|}
\hline Semana & Sesión 1 & Sesión 2 & Objetivos \\
\hline 7 & $\begin{array}{l}\text { Presentación del proyecto } \\
\text { Haptic Paddle }\end{array}$ & Ensamblado mecánico & Dispositivo ensamblado \\
\hline 8 & $\begin{array}{l}\text { Electrónica de control y co- } \\
\text { nexionado }\end{array}$ & $\begin{array}{l}\text { Conexionado y test del siste- } \\
\text { ma de control }\end{array}$ & $\begin{array}{l}\text { Ejecución de los programas } \\
\text { de test }\end{array}$ \\
\hline 9 & $\begin{array}{l}\text { Introducción a la programa- } \\
\text { ción del Haptic Paddle me- } \\
\text { diante simulink }\end{array}$ & $\begin{array}{l}\text { Programación de modelos } \\
\text { dinámicos }\end{array}$ & Ejemplo de muelle y fricción \\
\hline 10 & $\begin{array}{l}\text { Métodos y dispositivos de } \\
\text { calibración de par y lectura } \\
\text { de ángulo }\end{array}$ & $\begin{array}{l}\text { Programación de bloques } \\
\text { con magnitudes reales }\end{array}$ & $\begin{array}{l}\text { Bloques con magnitudes } \\
\text { reales }\end{array}$ \\
\hline 11 & $\begin{array}{l}\text { Diseño de proyectos. reglas, } \\
\text { ideas y limitaciones }\end{array}$ & Diseño de proyecto & prototipo de proyecto \\
\hline 12 & Proyecto & Documentación & $\begin{array}{l}\text { vídeos, programas (modelos) } \\
\text { y demostraciones }\end{array}$ \\
\hline
\end{tabular}

Tabla 3: Conjunto de proyectos diseñados por los diferentes grupos de prácticas.

\begin{tabular}{|l|l|l|}
\hline Gr. & Título & Objetivos \\
\hline 1 & Péndulo invertido & $\begin{array}{l}\text { Programación de la dinámica de un } \\
\text { péndulo invertido con visualización } \\
\text { gráfica bidimensional. } \\
\text { Programación de un sistema com- } \\
\text { puesto por dos haptic paddle donde } \\
\text { uno de ellos controla el lanzador y } \\
\text { otro la recepción en una plataforma. } \\
\text { Sistema de ejercicios con fuerzas } \\
\text { progresivas en secuencia para la rea- } \\
\text { lización controlada de ejercicios de } \\
\text { rehabilitación física. } \\
\text { Control de la posición unidimensio- } \\
\text { nal de una bola sobre una platafor- } \\
\text { ma pivotante con reflexión de fuer- } \\
\text { zas. } \\
\text { Juego en el que se hace botar verti- } \\
\text { calmente una pelota con una raque- } \\
\text { ta y visualización 3D. } \\
\text { Compensación de la dinámica de la } \\
\text { paleta y simulación de un péndulo } \\
\text { rígido con fuerzas externas. }\end{array}$ \\
5 & $\begin{array}{l}\text { Rehabilitación dentics } \\
\text { muñeca }\end{array}$ \\
\hline
\end{tabular}

laborativo.

La relativa simplicidad del dispositivo permite llevar a la práctica teorías de modelado de sistemas dinámicos y de control fácilmente, y su bajo coste permite desplegar un conjunto de dispositivos para poder crear grupos de uno o dos estudiantes por grupo. Asimismo permite que los estudiantes construyan sus propios haptics o que se lleven a casa los equipos.

Como valoración general, consideramos que se ha mejorado la docencia en la asignatura mediante unas herramientas atractivas para los alumnos, que les proporcionan unos conocimientos prácticos y teóricos que son comprobados mediante los resultados de los cuestionarios objetivos.

Las siguientes aplicaciones docentes de estos sistemas podrían encaminarse a su uso en otras asignaturas como Modelado y control o Control automático, donde pueden ser de gran ayuda al aprendizaje.

\section{Agradecimientos}

Este proyecto ha sido parcialmente financiado por el proyecto de Innovación educativa de la Universidad de Málaga PIE 15-180, por Seeed Studio (seeedstudio.com) e Ingeniería UNO (ingenieriauno.com).

Además, se agradece la colaboración de Sepehr Akbari Kalhor en los diseños de los prototipos, así como a todos los alumnos de la asignatura de Teleoperación y Telerrobótica del Máster de Mecatrónica.

\section{Referencias}

[1] Cabre, T. P., Cairol, M. T., Calafell, D. F., Ribes, M. T., and Roca, J. P. (2013). Projectbased learning example: controlling an educational robotic arm with computer vision. IEEE Revista Iberoamericana de Tecnologias del Aprendizaje, 8(3):135-142.

[2] Cappelleri, D. J. and Vitoroulis, N. (2013). The robotic decathlon: Project-based learning labs and curriculum design for an introductory robotics course. IEEE Transactions on Education, 56(1):73-81.

[3] Carbonaro, M., Rex, M., and Chambers, J. (2004). Using lego robotics in a project-based learning environment. The Interactive Multimedia Electronic Journal of Computer-Enhanced Learning, 6(1).

[4] Gandarias Palacios, J. M., Akbari Kalhor, S., and Gómez de Gabriel, J. M. (2016). Diseño y uso de una paleta háptica para prácticas de teleoperación con simulink.

[5] Gassert, R., Metzger, J.-C., Leuenberger, K., 
Popp, W. L., Tucker, M. R., Vigaru, B., Zimmermann, R., and Lambercy, O. (2013). Physical student-robot interaction with the ethz haptic paddle. IEEE Transactions on Education, 56(1):9-17.

[6] Gil, J., Muñoz, A., Torres, V., and Gómez, J. (2014). Uso de simulink y arduino para prácticas de robótica. XXXV Jornadas de Automática. Valencia.

[7] Gomez-de Gabriel, J. M., Mandow, A., Fernandez-Lozano, J., and Garcia-Cerezo, A. J. (2011). Using lego nxt mobile robots with labview for undergraduate courses on mechatronics. IEEE Transactions on Education, $54(1): 41-47$.

[8] Gómez de Gabriel, J. M., Muñoz Ramírez, A. J., and Fernández Lozano, J. J. (2015). Ingeniería basada en modelos en prácticas de robótica. XXXVI Jornadas de Automática. Bilbao.

[9] Gorlewicz, J. L., Kratchman, L. B., and WEBSTER III, R. J. (2014). Haptic paddle enhancements and a formal assessment of student learning in system dynamics. Advances in Engineering Education, 4(2).

[10] Gorlewicz, J. L. and Webster III, R. J. (2012). A formal assessment of the haptic paddle laboratories in teaching system dynamics. In American Society for Engineering Education. American Society for Engineering Education.

[11] Johnson, D., Johnson, R., and Johnson, E. (1999). Los nuevos círculos del aprendizaje La cooperación en el aula y la escuela. Aique.

[12] Maldonado Pérez, M. (2008). Aprendizaje basado en proyectos colaborativos. Una experiencia en educacion superior. Revista de Educación, 14(28).

[13] Martinez, M. O., Morimoto, T. K., Taylor, A. T., Barron, A. C., Pultorak, J. A., Wang, J., Calasanz-Kaiser, A., Davis, R. L., Blikstein, P., and Okamura, A. M. (2016). 3-d printed haptic devices for educational applications. In Haptics Symposium (HAPTICS), 2016 IEEE, pages 126-133. IEEE.

[14] Muñoz Ramírez, A. J. and Gomez De Gabriel, J. M. (2016). Modelar o programar en prácticas de robótica. XXXVII Jornadas de $A u$ tomática. Madrid.

[15] Okamura, A. M., Richard, C., Cutkosky, M., et al. (2002). Feeling is believing: Using a forcefeedback joystick to teach dynamic systems. Journal of Engineering Education, 91(3):345349 .

[16] Rose, C. G., French, J. A., and O'Malley, M. K. (2014). Design and characterization of a haptic paddle for dynamics education. In Haptics Symposium (HAPTICS), 2014 IEEE, pages 265-270. IEEE.

[17] Salinas, J. (2000). El aprendizaje colaborativo con los nuevos canales de comunicación. In Cabero, J., editor, Nuevas Tecnologías aplicadas a la Educación, pages 199-228. Síntesis.

[18] TaisLab. https://github.com/taislab/hapticpaddle. http://taislab.uma.es.

[19] TaisLab. Universidad de málaga. http://taislab.uma.es. 